\title{
Role of relaxation in the quantum measurement of a superconducting qubit using a nonlinear oscillator
}

\author{
T. Picot, ${ }^{1}$ A. Lupaşcu, ${ }^{2}$ S. Saito, ${ }^{3}$ C. J. P. M. Harmans, ${ }^{1}$ and J. E. Mooij ${ }^{1}$ \\ ${ }^{1}$ Kavli Institute of Nanoscience, Delft University of Technology, P.O. Box 5046, 2600GA Delft, The Netherlands \\ ${ }^{2}$ Laboratoire Kastler Brossel, ENS-24 rue Lhomond, 75005 Paris, France \\ ${ }^{3}$ NTT Basic Research Laboratories, NTT Corporation, 3-1 Morinosato-Wakamiya, Atsugi-shi 243-0198, Japan
}

(Received 8 September 2008; published 21 October 2008)

\begin{abstract}
We analyze the relaxation of a superconducting flux qubit during measurement. The qubit state is measured with a nonlinear oscillator driven across the threshold of bifurcation, acting as a switching dispersive detector. This readout scheme is of quantum nondemolition type. Two successive readouts are used to analyze the evolution of the qubit and the detector during the measurement. We introduce a simple transition rate model for characterizing the qubit relaxation and the detector switching process. Corrected for qubit relaxation the readout fidelity is at least 95\%. Qubit relaxation strongly depends on the driving strength and the state of the oscillator.
\end{abstract}

DOI: 10.1103/PhysRevB.78.132508

PACS number(s): 85.25.Cp, 03.67.Lx, 85.25.Dq

Superconducting qubits are quantum systems based on microfabricated superconducting circuits with one or more Josephson junctions as nonlinear elements. ${ }^{1}$ They are artificial quantum systems, with properties that can be defined by design of the mesoscopic parameters of the circuit. In superconducting qubits quantum state readout is of considerable interest since the fabricated nature of qubit and detector allows full control of the qubit-detector coupling strength. Consequently, aspects of quantum measurement can be experimentally investigated that are commonly not easy to access. These include the realization of high fidelity ${ }^{2}$ and projective $^{3,4}$ measurements, partial measurements, ${ }^{5}$ and the continuous observation of qubit dynamics. ${ }^{6,7}$ In addition, state readout is a subject relevant to quantum computing: projective measurements are an essential part of protocols for quantum information processing.

Qubit-state readout can be performed in various ways. In dispersive readout the qubit is coupled to an oscillator, with a quadratic type of interaction. As a result of this nonlinear coupling, the resonance frequency of the oscillator becomes qubit state dependent. ${ }^{7,8}$ The state of the qubit can thus be inferred from a measurement of the properties of the oscillator.

Nonlinear switching detectors are very attractive as they are able to amplify the information extracted from the qubit, leading to very fast readout with high fidelity. Here we present a detailed experimental analysis of switching dispersive readout of a superconducting flux qubit with a nonlinear oscillator. We introduce a simple model that allows one to characterize the detector switching process and the qubit relaxation induced by the operation of the detector. We find that the main source of measurement error is qubit relaxation induced by the operation of the detector.

The persistent current flux qubit circuit $^{9}$ is a superconducting loop interrupted by three Josephson junctions [see Fig. 1(a)]. Biased with an external flux $\Phi_{\mathrm{qb}}$ close to half a flux quantum $\Phi_{0} / 2$, it behaves as a quantum two-level system. The ground state $|g\rangle$ and the excited state $|e\rangle$ are quantum superpositions of two oppositely circulating persistent currents $\left( \pm I_{p}\right)$. In the basis of the current operator $\hat{I}=I_{p} \hat{\sigma}_{z}$,

the Hamiltonian of the flux qubit is $\hat{H}=\frac{1}{2}\left(\epsilon \hat{\sigma}_{z}+\Delta \hat{\sigma}_{x}\right)$, where $\epsilon=2 I_{p}\left(\Phi_{\mathrm{qb}}-\frac{1}{2} \Phi_{0}\right)$ and $\Delta$ is the quantum tunneling energy between the two current states. Here $\Delta / h=5 \mathrm{GHz}$ and the qubit is operated at a frequency $F_{\mathrm{qb}}=\sqrt{\epsilon^{2}+\Delta^{2}} / h=14.2 \mathrm{GHz}$.

Our detector is an oscillator formed by the inductance of a dc superconducting quantum interference device (SQUID) and a capacitor. The inductance of the SQUID and consequently the resonance frequency of the oscillator $F_{0}$ depend on the flux $\Phi_{\text {sq }}$ enclosed in the SQUID loop. The oscillator is operated at a frequency $F_{0}=1.5 \mathrm{GHz}$ and has a quality factor $Q=20$. The SQUID inductance is nonlinearly dependent on the SQUID current. Due to this nonlinearity, when the

a

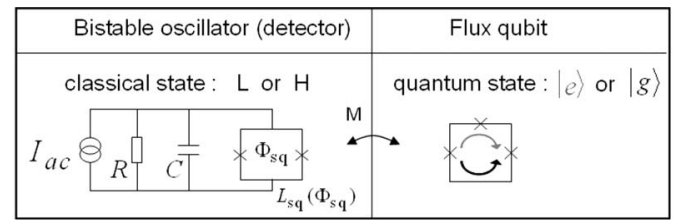

$$
\mathrm{b}
$$
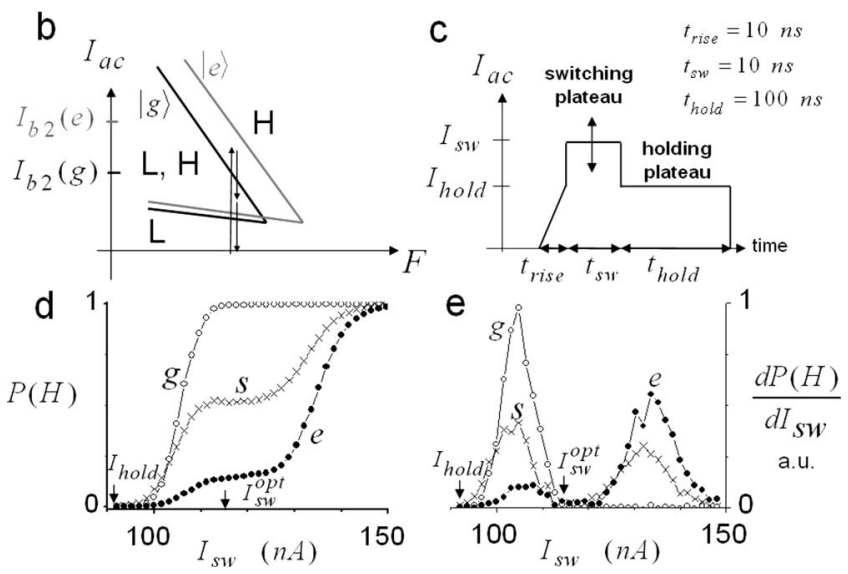

FIG. 1. (a) Qubit and readout circuit. (b) Bistability diagram of the oscillator for the two qubit states $|g\rangle$ and $|e\rangle$. (c) Oscillator driving amplitude for qubit readout. (d) Oscillator switching probability $P(H)$ as a function of the switching plateau driving amplitude $I_{\text {sw }}$ for the qubit prepared initially with a Rabi pulse, $0(g)$, $\pi(e)$, and $3 \pi / 2(s)$. (e) First derivative of $P(H)\left(I_{\mathrm{sw}}\right)$. 
oscillator is driven at a frequency $F<F_{0}\left(1-\frac{\sqrt{3}}{2 O}\right)$, it can switch between a state of low oscillation amplitude (labeled $L)$ and a state of high oscillation amplitude (labeled $H) .{ }^{10}$ Three situations are possible depending on the amplitude of the driving current $I_{\mathrm{ac}}$ compared to the two bifurcation currents $I_{b 1}(F)<I_{b 2}(F)$. For weak driving $I_{\mathrm{ac}}<I_{b 1}$ the oscillator is in the $L$ state. For strong driving $I_{\mathrm{ac}}>I_{b 2}$ the oscillator is in the $H$ state. For intermediate driving $I_{b 1}<I_{\mathrm{ac}}<I_{b 2}$, the oscillator is bistable and can be in either of the $L$ and $H$ states.

Due to the flux dependence of the SQUID inductance, the upper bifurcation current $I_{b 2}$ is highly sensitive to the flux $\Phi_{\text {sq. }}$. As the expectation value of the flux generated by the qubit is different for the two energy eigenstates, $I_{b 2}$ depends on the qubit state. In particular we choose the qubit bias such that $I_{b 2}(g)<I_{b 2}(e)$. For the readout of the flux qubit, the oscillator driving amplitude $I_{\mathrm{sw}}$ is increased to a value $I_{b 2}(\mathrm{~g})$ $<I_{\mathrm{sw}}<I_{b 2}(e)$ [see Figs. 1(b) and 1(c)], such that the oscillator switches to the $H$ state with a high probability if the qubit is in $|g\rangle$, while it stays in the $L$ state if the qubit is $|e\rangle$. This first time interval (the switching plateau) with duration $t_{\mathrm{sw}}$ constitutes the actual measurement interaction. To optimally discriminate between the two oscillator states $L$ and $H$, noise from the detection electronics needs to be averaged out. This is performed during the holding plateau $\left(t_{\text {hold }}\right)$ with the driving amplitude decreased such that both oscillator states can be maintained without switching or retrapping.

It should be noted that the large difference between $F_{\mathrm{qb}}$ and $F_{0}$ inhibits energy exchange between the qubit and the oscillator. The states $|e\rangle$ and $|g\rangle$ are thus preserved during the measurement.

As shown in Figs. 1(d) and 1(e), the two bifurcation currents $I_{b 2}(g)$ and $I_{b 2}(e)$ can be remarkably well resolved. The qubit readout is performed at an amplitude $I_{\mathrm{sw}}^{\mathrm{opt}}=115 \mathrm{nA}$, where the switching probability is $P^{g}(H)=99.7 \%$ if the qubit is in $|g\rangle$ and $P^{e}(H)=14.6 \%$ if the qubit is in $|e\rangle$, resulting in a readout contrast of $85 \%$. The main loss of readout contrast occurs when the qubit is in $|e\rangle$, suggesting that the readout fidelity is limited by the qubit relaxation before or during the measurement.

In practical flux qubits one finds a very irregular dependence of the relaxation on flux bias due to electromagnetic modes and "natural" two-level systems. The qubit is operated at a flux bias where the relaxation rate is locally minimal. During measurement, the SQUID transport current varies in time. By second-order processes, this oscillation shifts the average value of the circulating current, thus shifting the qubit bias. At that new point, relaxation is likely to be faster. More seriously, the qubit flux bias is swept at the oscillator driving frequency. In the $H$ state, the flux is modulated over a range as large as $5 \mathrm{~m} \Phi_{0}$ corresponding to a sweep of the energy splitting over $5 \mathrm{GHz}$. The qubit can thus be swept through regions where the relaxation rate is much higher. Moreover, when the oscillator is driven into its nonlinear regime, new channels of relaxation might open where qubit energy is transferred directly to the oscillator. ${ }^{11}$

We first measure the qubit relaxation under conditions where the oscillator is fixed in either the $L$ or the $H$ state. The qubit is initially prepared in $|e\rangle$. Next the oscillator is set either in the $H$ state or in the $L$ state. To prepare $H$ a short high driving pulse $I_{\mathrm{ac}}>I_{b 2}^{e}$ [dashed line in Fig. 2(a)] is ap-
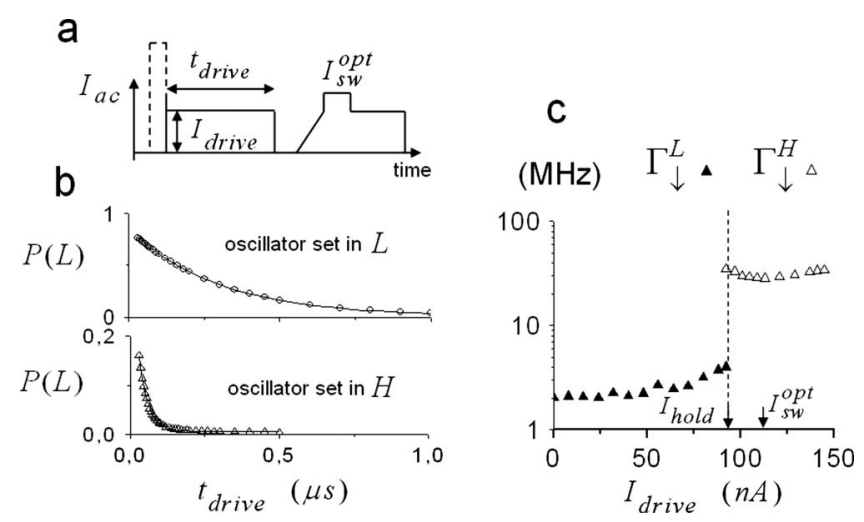

FIG. 2. (a) Oscillator driving sequence used to analyze the qubit relaxation. In the first driving pulse the oscillator is set in either $L$ or $H$ (see text). (b) Decay of the probability for the measurement outcome $L$ (due to the qubit relaxation). (c) Qubit relaxation rates versus the oscillator driving amplitude $I_{\text {drive }}$ for the oscillator in the state $L$ or $H$.

plied. Subsequently the oscillator is driven for a time $t_{\text {drive }}$ at an amplitude $I_{\text {drive }}$. Afterward the qubit state is read out with a regular measurement pulse. Figure 2(b) as an example gives the decay in time of the probability for readout in the $L$ state when the oscillator is driven with an amplitude $I_{\text {drive }}$ $=I_{\text {hold }}$. This decay is exponential and is due to qubit relaxation. The two qubit relaxation rates $\Gamma^{L}$ and $\Gamma^{H}$ for the two states of the oscillator are significantly different. Figure 2(c) shows the dependence of the qubit relaxation rates on the oscillator driving amplitude for $I_{\text {drive }}<I_{\text {hold }}$ and $I_{\text {drive }}>I_{\text {hold }}$ when the oscillator is in the $L$ or $H$ state, respectively. $\Gamma_{\downarrow}^{H}$ is almost constant, whereas $\Gamma_{\downarrow}^{L}$ increases with the driving amplitude.

Similarly, we characterize the qubit relaxation during the rising part of the readout pulse with the effective relaxation rate $\Gamma_{\downarrow}^{\text {rise }}$ obtained from an exponential decay fitting of $P(L)$ as a function of the rise time $t_{\text {rise }}$. Corrected for the relaxation during the rising part of the readout pulse $(5 \%)$ and initial qubit preparation errors $(5 \%),{ }^{12}$ the readout fidelity is $f$ $=95 \%$. The remaining errors occur during the switching plateau.

The approach used so far is well suited for analyzing the qubit relaxation for a driving amplitude at which the oscillator is in a stable state. However for higher driving amplitude $I_{\text {drive }}>I_{\text {hold }}$ and especially at the driving amplitude during the switching plateau, the $L$ state is metastable and can switch to the $H$ state. Therefore to analyze the qubit relaxation, the oscillator switching process needs to be included.

In the following we analyze the oscillator switching and the qubit relaxation during the switching plateau. The solid line in Fig. 3(b) shows the oscillator switching probability when the qubit is in $|g\rangle$ as a function of the duration of the switching plateau $t_{\mathrm{sw}}$. We distinguish two regimes, indicated as I and II. The boundary of the two regimes is after a time of about $10 \mathrm{~ns}$, indicated as $t_{\mathrm{I} / \mathrm{II}}$. In regime I the switching probability increases very fast. At the origin of the switching plateau, the oscillator driving amplitude is increased in about $1 \mathrm{~ns}$, probably leading to nonadiabatic effects. In regime II the switching probability increases with a constant rate. The 


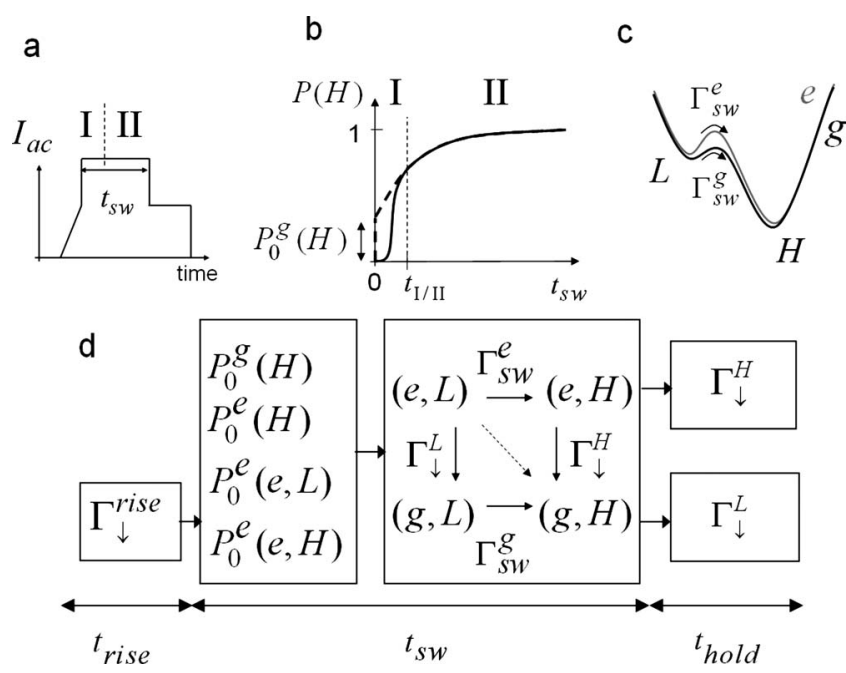

FIG. 3. (a) Oscillator driving amplitude. (b) Switching probability as a function of the duration of the switching plateau (solid line). (c) Effective potential barrier between $L$ and $H$, for the qubit state $|g\rangle$ or $|e\rangle$. (d) Schematic of the model used to describe the evolution of the qubit and the oscillator during the readout pulse, including qubit relaxation rates $\Gamma_{\downarrow}^{L}$ and $\Gamma_{\downarrow}^{H}$ and oscillator switching rates $\Gamma_{\mathrm{sw}}^{e}$ and $\Gamma_{\mathrm{sw}}^{g} \cdot P_{0}^{g}(H), P_{0}^{e}(H), P_{0}^{e}(e, H)$, and $P_{0}^{e}(e, L)$ describe the regime I (see text).

switching rate $\Gamma_{\text {sw }}$ from the $L$ state to the $H$ state depends on the height of the effective potential barrier ${ }^{13}$ between $L$ and $H: \Delta U=U_{0}\left[1-\left(I_{\mathrm{ac}} / I_{b 2}\right)^{2}\right]^{3 / 2}$. For a driving current $I_{\mathrm{ac}}$ close to the upper bifurcation current $I_{b 2}$, the oscillator switching rate $\Gamma_{\mathrm{sw}}$ increases strongly by a few orders of magnitude. As $I_{b 2}$ is different for the two qubit states, $\Gamma_{\mathrm{sw}}$ strongly depends on the qubit state, which is the principle of the measurement. As $I_{b 2}(g)<I_{b 2}(e)$, it follows that $\Gamma_{\mathrm{sw}}^{g} \ll \Gamma_{\mathrm{sw}}^{e}$ [Fig. 3(c)].

We describe the qubit and the oscillator as a four-state system $(g, L),(g, H),(e, L)$, and $(e, H)$, with four corresponding occupation probabilities. Due to normalization, only three probabilities are independent. We use $P(H)$ $=P(e, H)+P(g, H), P(e, H)$, and $P(e, L)$. In regime II, oscillator switching $(L \rightarrow H)$ and qubit relaxation $(e \rightarrow g)$ are described with the set of four rates $\Gamma_{\mathrm{sw}}^{g}, \Gamma_{\mathrm{sw}}^{e}, \Gamma_{\downarrow}^{L}$, and $\Gamma_{\downarrow}^{H}[\mathrm{Fig}$. 3(d)]. Oscillator retrapping $(H \rightarrow L)$ and qubit excitation $(g$ $\rightarrow e)$ are negligible. The evolution of the occupation probabilities is given by

$$
\begin{gathered}
\frac{d P(e, L)}{d t}=-P(e, L)\left(\Gamma_{\mathrm{sw}}^{e}+\Gamma_{\downarrow}^{L}\right), \\
\frac{d P(e, H)}{d t}=P(e, L) \Gamma_{\mathrm{sw}}^{e}-P(e, H) \Gamma_{\downarrow}^{H}, \\
\frac{d P(g, L)}{d t}=P(e, L) \Gamma_{\downarrow}^{L}-P(g, L) \Gamma_{\mathrm{sw}}^{g}, \\
\frac{d P(g, H)}{d t}=P(g, L) \Gamma_{\mathrm{sw}}^{g}+P(e, H) \Gamma_{\downarrow}^{H} .
\end{gathered}
$$
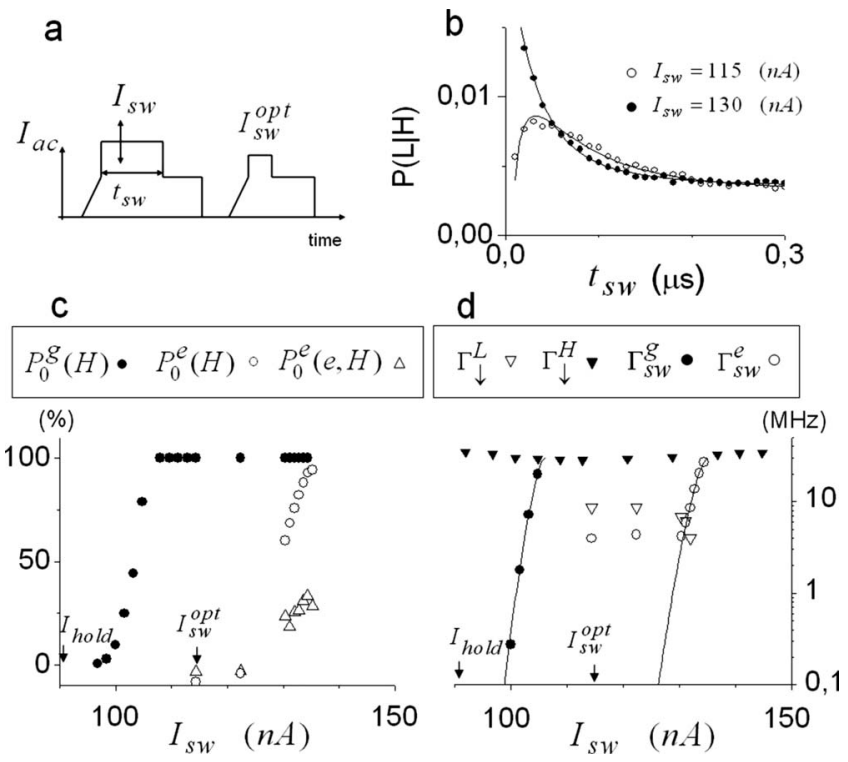

FIG. 4. (a) Sequence of two successive readouts used to analyze the qubit state after the first measurement. (b) Conditional probability for the measurement outcome $L$ after a switching event in the first readout. (c) $P_{0}^{g}(H), P_{0}^{e}(H)$, and $P_{0}^{e}(e, H)$. (d) Oscillator switching rates $\Gamma_{\mathrm{sw}}^{e}$ and $\Gamma_{\mathrm{sw}}^{g}$, qubit relaxation rates $\Gamma_{\downarrow}^{L}$ and $\Gamma_{\downarrow}^{H}$ as a function of the switching plateau driving amplitude $I_{\mathrm{sw}}$.

For a given initial qubit state $i(g$ or $e$ ) at the origin of the switching plateau $t=0$, we denote $P_{0}^{i}(H), P_{0}^{i}(e, H)$, and $P_{0}^{i}(e, L)$ as the initial conditions for the set of equations (1). They quantify the evolution of the qubit and the oscillator evolution during regime I. We choose to define the initial conditions at $t=0$ and not at $t=t_{\mathrm{I} / \mathrm{II}}$. They are obtained by extrapolating $P(H), P(e, H)$, and $P(g, L)$ in regime II to $t=0$. In the more general case of an initial qubit state with an occupation probability of $|e\rangle, P(e)=x$, the initial conditions are $P_{0}(H)=x P_{0}^{e}(H)+(1-x) P_{0}^{g}(H)$ [and similarly for $P_{0}(e, H)$ and $\left.P_{0}(e, L)\right]$.

If the qubit is initially in $|g\rangle$, the only relevant rate is $\Gamma_{\mathrm{sw}}^{g}$. The set of equations (1) reduces to $d P(H) / d t$ $=[1-P(H)] \Gamma_{\mathrm{sw}}^{g}$. For a given switching plateau driving amplitude $I_{\mathrm{sw}}, P_{0}^{g}(H)$ and $\Gamma_{\mathrm{sw}}^{g}$ are extracted by fitting the switching probability as a function of $t_{\mathrm{sw}}$ with $P(H)$ $=1-\left[1-P_{0}^{g}(H)\right] \exp \left(-t_{\mathrm{sw}} \Gamma_{\mathrm{sw}}^{g}\right)$ [as shown by the dashed line in Fig. 3(b)].

The driving amplitude $I_{\mathrm{sw}}^{\mathrm{opt}}$ used for the measurement is higher than $I_{b 2}(g)$. In that case the $L$ state does not exist when the qubit is in $|g\rangle$, so qubit relaxation directly causes oscillator switching. The state $(g, L)$ is eliminated from the set of equations (1) by assuming that $\Gamma^{L}$ corresponds to the transition $(e, L) \rightarrow(g, H)$ shown by the dashed arrow in Fig. $3(\mathrm{~d})$. If the qubit is in $|e\rangle$, two switching processes are possible, either due to qubit relaxation (rate $\Gamma_{\downarrow}^{L}$ ) or to switching while the qubit is excited (rate $\Gamma_{\mathrm{sw}}^{e}$ ). The sum $\Gamma_{\mathrm{sw}}=\Gamma_{\downarrow}^{L}+\Gamma_{\mathrm{sw}}^{e}$ and $P_{0}^{e}(H)$ can be extracted from $P(H)$, given by $P(H)=1-x\left[1-P_{0}^{e}(H)\right] \exp \left(-t_{\mathrm{sw}} \Gamma_{\mathrm{sw}}\right)$, where $x$ is the occupation probability of $|e\rangle$ at the origin of the switching plateau. ${ }^{14}$ Depending on the process, the state of the qubit after switching is either $|g\rangle$ or $|e\rangle$. Therefore, it is possible to discriminate between the two processes by measuring the qubit state 
after a switching event. At the end of the switching plateau, $P(e, H)$ is given by

$$
\begin{aligned}
P(e, H)\left(t_{\mathrm{sw}}\right)= & x P_{0}^{e}(e, H) e^{-t_{\mathrm{sw}} \Gamma_{\downarrow}^{H}} \\
& +x\left[1-P_{0}^{e}(H)\right] \frac{\Gamma_{\mathrm{sw}}^{e}}{\Gamma_{\downarrow}^{H}-\Gamma_{\mathrm{sw}}}\left[e^{-t_{\mathrm{sw}} \Gamma_{\mathrm{sw}}}-e^{-t_{\mathrm{sw}} \Gamma_{\downarrow}^{H}}\right] .
\end{aligned}
$$

If relaxation is the only process which can lead to oscillator switching, then $P(e, H)=0$. In Eq. (2), the first term proportional to $P_{0}^{e}(e, H)$ corresponds to switching events during regime $\mathrm{I}$, whereas the second term proportional to $\Gamma_{\mathrm{sw}}^{e}$ corresponds to switching events during regime II.

The occupation probability of $|e\rangle$ after a switching event, given by the conditional probability $P(e \mid H)=P(e, H) / P(H)$, is measured with a second, successive readout. The conditional probability $P(L \mid H)$ for a measurement outcome $L$ after a switching event is $P(L \mid H)=A P(e \mid H)+B$, where $A$ $=0.9 \exp \left(-t_{\text {hold }} \Gamma_{\downarrow}^{H}\right)$ includes the contrast of the second readout and the qubit relaxation, and $B=3.5 \times 10^{-3}$ is the finite measurement error when the qubit is in $|g\rangle$. When the oscillator is in the $H$ state, qubit relaxation during the holding plateau is very strong. Therefore, the remaining signal is very small, $P(L \mid H) \approx 10^{-2}$. A key point for our analysis is the very high contrast of the readout allowing a resolution of $P(L \mid H)$ as small as $10^{-3} . \Gamma_{\mathrm{sw}}^{e}$ and $P_{0}^{e}(e, H)$ are extracted from a fit of $P(e, H)$ to Eq. (2). The decay rates $\Gamma_{\mathrm{sw}}$ and $\Gamma_{\downarrow}^{H}$ are fixed (extracted previously), and $P_{0}^{e}(e, H)$ and $\Gamma_{\mathrm{sw}}^{e}$ are fitting parameters. The obtained values of $P_{0}^{e}(e, H), \Gamma_{\mathrm{sw}}^{e}$, and $\Gamma_{\downarrow}^{L}$ are shown in Figs. 4(c) and 4(d). For comparison, $P_{0}^{g}(H), P_{0}^{e}(H)$, $\Gamma_{\mathrm{sw}}^{g}$, and $\Gamma_{\downarrow}^{H}$ obtained previously are shown as well.

The ratio $\Gamma_{\mathrm{sw}}^{e} / \Gamma_{\mathrm{sw}}^{g}$ determines the intrinsic readout fidelity. The solid black lines in Fig. 4(d) are fits of $\Gamma_{\mathrm{sw}}^{e}$ and $\Gamma_{\mathrm{sw}}^{g}$ using the oscillator escape rate equation as given in Ref. 13, from which we extract $I_{b 2}(g)=108 \mathrm{nA}$ and $I_{b 2}(e)=136 \mathrm{nA}$. Extrapolating $\Gamma_{\mathrm{sw}}^{e}$, the intrinsic readout fidelity would be $99.9 \%$. However, in practice we observe a saturation of $\Gamma_{\mathrm{sw}}^{e}$ at approximately $4 \mathrm{MHz}$.

The qubit relaxation rate $\Gamma_{\downarrow}^{L}$ can directly limit the readout fidelity. The independent determination of $\Gamma_{\downarrow}^{L}$ and $\Gamma_{\mathrm{sw}}^{e}$ is an important result of this Brief Report. At the driving amplitude $I_{\mathrm{sw}}^{\mathrm{opt}}$ used for the measurement, $\Gamma_{\downarrow}^{L}$ is higher than $\Gamma_{\mathrm{sw}}^{e}$. Therefore, for measurement times beyond the oscillator transient period, qubit relaxation is the main process limiting the readout fidelity.

To summarize, we have introduced a simple model for characterizing the qubit relaxation and the oscillator switching process during measurement of a flux qubit. Qubit relaxation increases significantly with increasing driving strength with the oscillator in the low-amplitude state. It jumps to a much higher rate when the oscillator switches to its highamplitude state. Corrected for qubit relaxation and initial qubit preparation errors, the readout fidelity is at least $95 \%$.

We thank F. Wilhelm, I. Şerban, S. Ashhab, Y. Nazarov, and $\mathrm{H}$. Wei for useful discussions. This work was supported by the Dutch Organization for Fundamental Research on Matter (FOM), EU EuroSQIP, the EU Marie Curie program, and the NanoNed program.
${ }^{1}$ M. Devoret, A. Wallraff, and J. Martinis, arXiv:cond-mat/ 0411174 (unpublished).

${ }^{2}$ A. Lupaşcu, E. F. C. Driessen, L. Roschier, C. J. P. M. Harmans, and J. E. Mooij, Phys. Rev. Lett. 96, 127003 (2006).

${ }^{3}$ A. Lupaşcu, S. Saito, T. Picot, P. C. de Groot, C. J. P. M. Harmans, and J. E. Mooij, Nat. Phys. 3, 119 (2007).

${ }^{4}$ N. Boulant, G. Ithier, P. Meeson, F. Nguyen, D. Vion, D. Esteve, I. Siddiqi, R. Vijay, C. Rigetti, F. Pierre, and M. Devoret, Phys. Rev. B 76, 014525 (2007).

${ }^{5}$ N. Katz, M. Ansmann, R. C. Bialczak, E. Lucero, R. McDermott, M. Neeley, M. Steffen, E. M. Weig, A. N. Cleland, J. M. Martinis, and A. Korotkov, Science 312, 1498 (2006).

${ }^{6}$ A. N. Korotkov, Phys. Rev. B 60, 5737 (1999).

${ }^{7}$ E. Il'ichev, N. Oukhanski, A. Izmalkov, Th. Wagner, M. Grajcar, H.-G. Meyer, A. Yu. Smirnov, A. Maassen van den Brink, M. H. S. Amin, and A. M. Zagoskin, Phys. Rev. Lett. 91, 097906
(2003).

${ }^{8}$ A. Lupaşcu, C. J. M. Verwijs, R. N. Schouten, C. J. P. M. Harmans, and J. E. Mooij, Phys. Rev. Lett. 93, 177006 (2004).

${ }^{9}$ J. E. Mooij, T. P. Orlando, L. Levitov, L. Tian, C. H. van der Wal, and S. Lloyd, Science 285, 1036 (1999).

${ }^{10}$ I. Siddiqi, R. Vijay, F. Pierre, C. M. Wilson, M. Metcalfe, C. Rigetti, L. Frunzio, and M. H. Devoret, Phys. Rev. Lett. 93, 207002 (2004).

${ }^{11}$ I. Serban, Ph.D. thesis, Ludwig-Maximilians University, 2008.

${ }^{12}$ Taking into account the period $T=3.2 \mathrm{~ns}$ and the decay time $\tau$ $=15 \mathrm{~ns}$ of the Rabi oscillations, we estimate the preparation fidelity of $|e\rangle$ to be $95 \%$.

${ }^{13}$ M. I. Dykman, Physica (Amsterdam) 104A, 480 (1980).

${ }^{14}$ Due to the initial preparation errors of $5 \%$ and the qubit relaxation during the rising part of the readout pulse of $5 \%, x=0.90$. 